\section{The successful treatment of inflammatory aortitis in \\ Behçet's disease with Infliximab and endovascular intra-aortic stenting}

\author{
Martin A. Lee, ${ }^{1}$ Stuart Kyle, ${ }^{2}$ \\ Robert J. Moots 3
}

'Rheumatology Department, National Hospital of Rheumatic Diseases, Bath; 2Rheumatology Department, North Devon District Hospital, Barnstaple; ${ }^{3}$ Clinical Sciences Centre, University Hospital Aintree, Liverpool, UK

\begin{abstract}
Behçet's disease is a systemic vasculitis of unknown aetiology that may involve veins and arteries of all sizes. It occurs most commonly in the second or third decades of life and it is characterised by recurrent oral and genital ulceration and chronic relapsing uveitis. We present here a case of Behçet's disease complicated by an inflammatory aortitis successfully treated with infliximab and endovascular stenting.
\end{abstract}

\section{Introduction}

Behçet's disease is a systemic vasculitis of unknown aetiology that may involve veins and arteries of all sizes. The disease occurs most commonly in the second or third decades of life and is characterised by recurrent oral and genital ulceration and chronic relapsing uveitis. Other features of the disease include pathergy, erythema nodosum, arthritis, venous thrombosis, arteritis, gastrointestinal ulceration and neurological involvement. There have been a number of trials, case series and case reports demonstrating the efficacy of treatment with infliximab (a chimeric monoclonal antibody to soluble and cell bound tumour necrosis factor- $\alpha$ ) for a number of complications of Behçet's disease including uveitis, intestinal involvement, neurological involvement, resistant oral ulceration, and hepatic vein thrombosis. ${ }^{1-7}$ However, there is currently a lack of literature surrounding the use of infliximab for Behçet's-related inflammatory aortitis. We present a case of Behçet's disease complicated by an inflammatory aortitis successfully treated with infliximab and endovascular stenting.

\section{Case Report}

A 27-year-old Caucasian woman was referred to rheumatology for an in-patient review in June 2009 after the birth of her second child. She had suffered an episode of post-puerperal psychosis. It was difficult to obtain a clear history due to the patient appearing confused. She gave a history of oral and genital ulceration although also gave a history of herpes simplex genital infection. She gave no history of eye problems or pathergy. On further review of her notes, she had suffered an episode of post-puerperal psychosis after the birth of her first child and had previously been documented as having a skin lesion consistent with erythema nodosum. She had previously been diagnosed with a renal vein thrombosis in another hospital whilst on holiday and had also undergone a colonoscopy with biopsy due to diarrhoea. The biopsy result was consistent with an inflammatory colitis. The diagnosis of Behçet's disease had been questioned in the past but her symptoms had abated. On examination as an inpatient, there was no evidence of oral or genital ulceration. Her C-reactive protein (CRP) was raised at $50 \mathrm{mg} / \mathrm{L}$ (normal range $<5 \mathrm{mg} / \mathrm{L}$ ) and her autoimmune profile was negative. A magnetic resonance imaging scan of her brain without contrast demonstrated a small white matter lesion which was reported as non-significant. It was concluded that there was no evidence of active Behçet's disease.

In August 2009, the patient with re-admitted with confusion, diarrhoea and weight loss. On examination the patient did not engage in conversation. There was a small nodular lesion on her left thigh and she was tender over the right iliac fossa. There was no organomegaly and peripheral pulses were present. There was no evidence of oral or genital ulceration. Blood tests revealed a raised CRP and alkaline phosphatase at $91 \mathrm{mg} / \mathrm{L}$ and $318 \mathrm{iu} / \mathrm{L}$ (normal range 40-140 iu/L) respectively. At the time a provisional diagnosis of a flare of inflammatory bowel disease was made and an abdominal ultrasound was requested to investigate the raised alkaline phosphatase. The ultrasound demonstrated an abnormally dilated aorta and a subsequent computed tomography (CT) scan demonstrated a hepatic vein thrombosis and an infra-renal aortic aneurysm which had leaked posteriorly but had sealed off (Figure 1).

A diagnosis of Behçet's disease with inflammatory aortitis was made and the patient was treated with $1 \mathrm{~g}$ intravenous (IV) methylprednisolone daily for 3 days followed by $40 \mathrm{mg}$ oral prednisolone daily. Her CRP responded well to the IV methylprednisolone. Based on discussion with a national expert, the patient was administered infliximab $5 \mathrm{mg} / \mathrm{kg}$ and transferred to a local centre with expertise in vascular surgery. She underwent successful
Correspondence: Martin A. Lee, Rheumatology Department, National Hospital of Rheumatic Diseases, Flat 43 Mills Bakery, Royal William Yard, Plymouth, PL1 3GD, UK.

Tel. +44.7799.450793.

E-mail: martinlee@doctors.org.uk

Key words: Behçet's disease, inflammatory aortitis, infliximab, endovascular stenting.

Received for publication:4 September 2011. Revision received: 13 February 2012.

Accepted for publication: 15 February 2012.

This work is licensed under a Creative Commons Attribution NonCommercial 3.0 License (CC BYNC 3.0).

CC Copyright M.A. Lee et al., 2012

Licensee PAGEPress, Italy

Rheumatology Reports 2012; 4:e2

doi:10.4081/rr.2012.e2

endovascular intra-aortic stenting through her right femoral artery. The patient was found to be HLA B51 negative and was warfarinised based upon her history of renal and hepatic vein thrombosis and stenting with a target INR (International normalized ratio) between 2.5 and 3.5. A repeat abdominal CT scan showed a

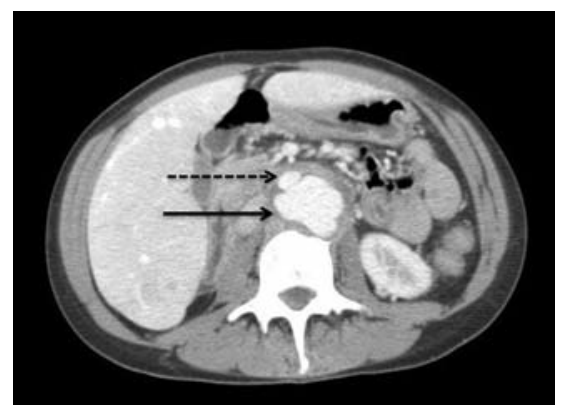

Figure 1. Axial compted tomography section through the abdomen demonstrating an inflammatory infra-renal false aortic aneurysm (arrow) separate from the true lumen (broken arrow).

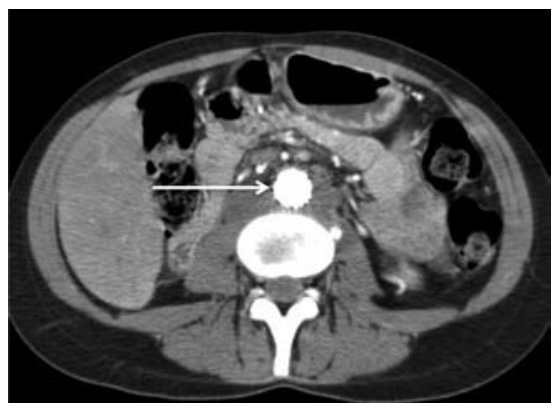

Figure 2. Axial computed tomography section through the abdomen post infliximab and endovascular intra-aortic stenting demonstrating an improvement in the inflammatory peri-aortic mass (arrow). 
marked improvement in the inflammatory peri-aortic mass (Figure 2).

It was decided to treat the patient with 5 $\mathrm{mg} / \mathrm{kg}$ infliximab at weeks 0,2 and 6 and 8 weekly thereafter as per the rheumatoid arthritis regime. Two weeks after the original infliximab infusion, the patient's condition was improving and her CRP had fallen to 35 $\mathrm{mg} / \mathrm{L}$. Her prednisolone dose was tapered down from $40 \mathrm{mg}$ daily. Three months after the original infliximab infusion, the patient was mentally alert and actively interested in her condition. She described herself as back to normal.

\section{Discussion}

Behçet's disease is an idiopathic inflammatory systemic vasculitis that may affect veins and arteries and is characterised by recurrent oral and genital ulceration. Tumour necrosis factor$\alpha$ (TNF- $\alpha$ ) is a pro-inflammatory cytokine that is thought to play a role in a number of inflammatory conditions such as rheumatoid arthritis and Behçet's disease. Infliximab is a chimeric monoclonal antibody to soluble and cell bound TNF- $\alpha$ that has profound anti-inflammatory effects. Infliximab has been used for a number of complications of Behçet's disease such as chronic relapsing uveitis. A number of systemic immunosuppressive agents such as corticosteroids, azathioprine and cyclophosphamide have been used in the treatment of major vessel involvement in Behçet's disease but there is no firm evidence to guide the management of this complication. ${ }^{8}$ Infliximab is administered intra- venously and therefore may have a rapid antiinflammatory effect on vessel walls. It may prove to be a useful therapy for life-threatening inflammatory complications of Behçet's disease and other systemic vasculitides. The patient in this case report was co-administered methylprednisolone and infliximab. The anti-inflammatory effects of methylprednisolone may have augmented the effects of infliximab in reducing the patient's peri-aortic inflammatory mass.

\section{Conclusions}

We describe an interesting case of Behçet's disease in a 27-year-old Caucasian female with a history of oral and genital ulceration, renal vein thrombosis and inflammatory colitis. She presented after 2 episodes of post-puerperal psychosis with a hepatic vein thrombosis and an inflammatory aortitis. Although endovascular stenting has been documented for aortic and arterial aneurysms in Behçet's disease, ${ }^{9}$ this is the first documented case in the literature of a Behçet's-related inflammatory aortitis treated successfully with infliximab and endovascular stenting.

\section{References}

1. Almoznino G, Ben-Chetrit E. Infliximab for the treatment of resistant oral ulcers in Behçet's disease: a case report and review of the literature. Clin Exp Rheumatol
2007;25:S99-102.

2. Seyahi E, Hamuryudan V, Hatemi G, et al. Infliximab in the treatment of hepatic vein thrombosis (Budd Chiari syndrome) in three patients with Behçet's syndrome. Rheumatology 2007; 46:1213-4.

3. Pipitone N, Olivieri I, Padula A, et al. Infliximab for the treatment of NeuroBehçet's disease: a case series and review of the literature. Arthritis Rheum 2008; 59:285-90.

4. Naganuma M, Sakuraba A, Hisamatsu T, et al. Efficacy of infliximab for induction and maintenance of remission in intestinal Behçet's disease. Inflamm Bowel Dis 2008;14:1259-64.

5. Al-Rayes H, Al-Swailem R, Al-Balawi M, et al. Safety and efficacy of infliximab therapy in active Behçet's uveitis: An open-label trial. Rheumatol Int 2008;29:53-7.

6. Olivieri I, Padula A, Leccese P, et al. Longlasting remission of severe Behçet's disease after the end of infliximab therapy. $\mathrm{J}$ Rheumatol 2009;36:855

7. Iwata S, Saito K, Yamaoka K. Effects of anti-TNF-alpha antibody infliximab in refractory entero-Behcets disease. Rheumatology 2009;48:1012-3.

8. Hatemi G, Silman A, Bang D, et al. EULAR recommendations for the management of Behcet disease. Ann Rheum Dis 2008;67: 1656-62.

9. Park JH, Chung JW, Joh JH, et al. Aortic and arterial aneurysms in Behçet's disease: Management with stent-grafts - initial experience. Radiology 2001;220:74550 . 